\title{
THE INFLUENCE OF VARYING As-Vs INTERVALS ON SPLIT FIRST HEART SOUNDS: ITS BEARING ON THE CAUSE OF SPLIT SOUNDS AND THE MECHANISM OF THE FIRST SOUND ${ }^{1}$
}

\author{
By CHARLES C. WOLFERTH AND ALEXANDER MARGOLIES \\ (From the Robinette Foundation, Medical Clinic, Hospital of the University of Pennsylvania, \\ Philadelphia)
}

(Received for publication May 15, 1935)

It has long been recognized that the first heart sound may include two major components (1). Under certain circumstances these two components may be found on auscultation to be separated so that the sound becomes split or reduplicated, a phenomenon that can be recorded by methods for registering heart sounds. Splitting of the first sound is present in a certain proportion of patients with either left or right complete bundle branch block (2) and in some with lesser grades of intraventricular conduction defect. (Unpublished observations, Wolferth and Margolies.) It is also present in many individuals with presumably healthy hearts (3). There tends to be a phasic respiratory incidence in the width of the split which is particularly marked in the presumably healthy individuals. Thus, in some cases, if the heart sounds are recorded throughout cycles of respiration, the first sound may be found split during certain phases of the cycle, and single during certain other phases (Figure 1). With an adequately damped apparatus for registration, the split components are recorded as a series of vibrations of short duration. In a case with phasic incidence of splitting the single first sounds are nearly always of considerably longer duration than either component of split sounds. Furthermore, if the duration of either component of a split sound is compared with the duration of the first sound in a case which shows no splitting, the duration of the latter is usually greater. This would suggest that even when there is no determinable splitting of the first sound both components may be present and at least partially merged.

The first step in the study of the mechanism of the first sound should be an attempt to discover what causes this duality of the sound. One gets

1 Presented in abstract before the American Society for Clinical Investigation May 6, 1935. but little help from the literature. Some writers have maintained that in split sounds, one component arises in the left and the other in the right ventricle (4). Others have contended that one component is muscular and the other valvular in origin. Thus Wiggers (5) states: "It is generally accepted ... that the first sound is fundamentally due to vibrations arising from: $(a)$ the friction of the interlacing bands of contracting ventricular muscle, and $(b)$ the closure of the A-V valves." He comments, however, that such an explanation accounts only for the main crescendo vibrations and leaves out of consideration both the introductory and final vibrations of the first sound and does not account for the second components of the aortic first sound. He found that vibrations recorded directly from different regions of the right and left ventricles show no essential differences. Hence, he states, they may be referred to in common as the ventricular sounds.

Recently, in the course of a study of time relations of various events dependent on right and left ventricular contraction, undertaken in the effort to discover the side of the significant lesion in the common type of bundle branch block (6), we have obtained some data that bear on the mechanism of split first sounds. In the few cases studied with widely split first sounds, asynchronism was found in ejection from the right and left ventricles, which corresponded roughly to the degree of asynchronism between the two major components of the sound. ${ }^{2}$ Furthermore, the studies indicated that in these cases with widely split first sounds, the first component began before ejection from either ventricle had begun and

\footnotetext{
2 Asynchronism in ejection from the two ventricles had previously been noted by Katz (7) in an experimental study on dogs. No attempt, however, was made to correlate this finding with the heart sounds.
} 

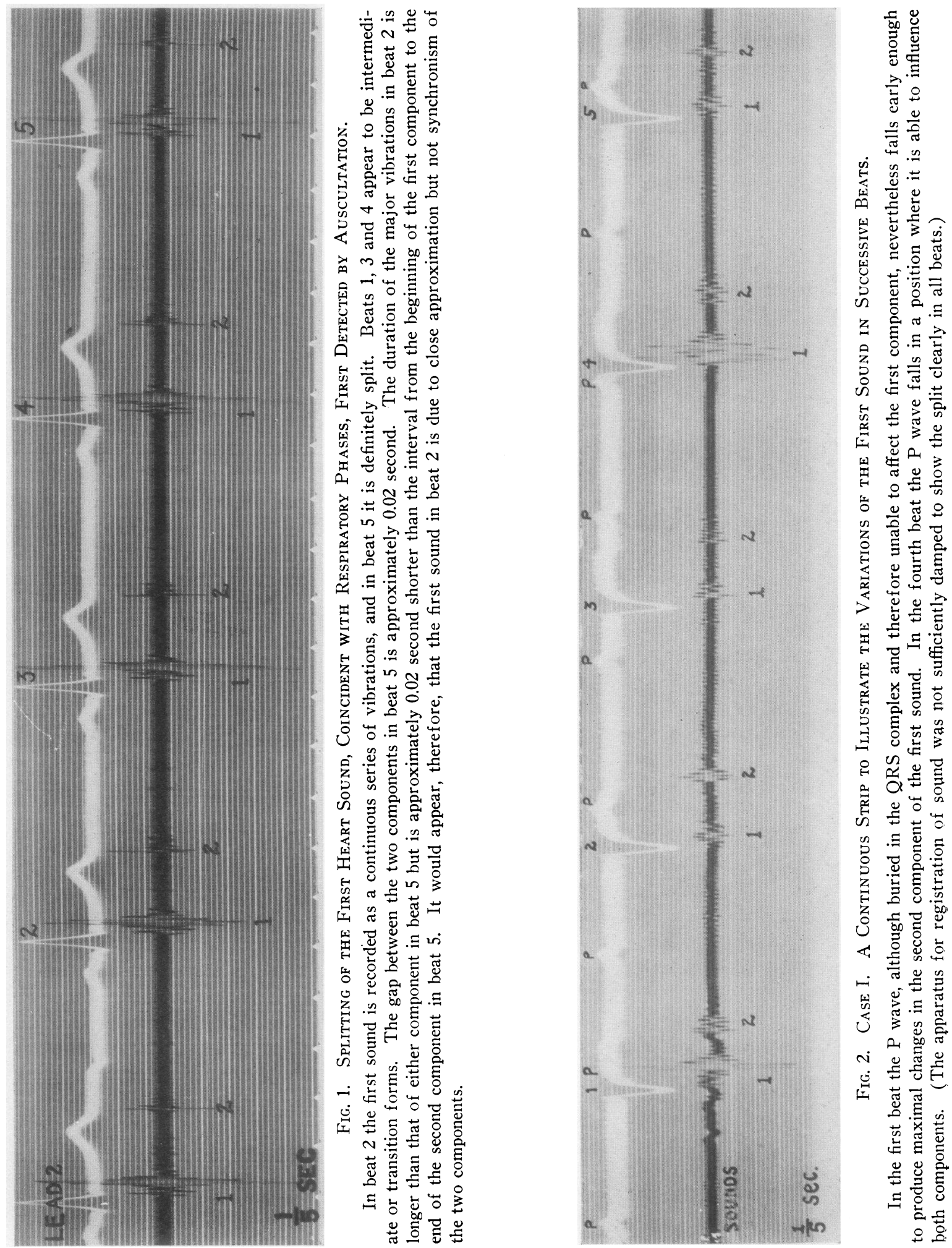
the second component usually after ejection from one ventricle had begun but always before ejection from the other. ${ }^{3}$ These correlations were regarded as pointing toward the hypothesis that, at least in certain cases, one of the major components of sound was contributed by the left ventricle and the other by the right ventricle. The significance of the evidence was discussed in the paper referred to (6).

The methods used to time the beginning of right and left ventricular ejection are accurate enough to reveal the presence of significant asynchronism in cases with widely split first sounds. However, in cases with slight splitting or merely prolongation of the first sound the possible errors in the measurements are sufficient to render the results of doubtful value. In view of the importance of establishing the nature of the duality of the first sound it seemed desirable to find some other method of testing the validity of the hypothesis.

It has long been known that in patients with complete heart block there may be marked variation in the loudness of the first sound from beat to beat. Griffith (9), who was the first to point out this phenomenon, concluded that the loud beats tended to occur when the auricles and ventricles beat at about the same time. Several years ago we made a study of this phenomenon and found that the As-Vs time relationship (as measured by the $\mathrm{P}-\mathrm{R}$ relation) exerts a remarkable influence on the amplitude of recorded vibrations of the first heart sound not only in complete heart block but also in other conditions (10). Furthermore, it could be demonstrated that the marked variations in amplitude corresponded to variations in loudness of the sounds recognizable by auscultation. Slight changes in As-Vs time were sometimes found to be associated with marked variations in the first sound. As a matter of fact, among the various factors governing loudness of

\footnotetext{
${ }^{3}$ Wiggers (8) states that the main vibrations of the first sound begin precisely with the onset of the rise of pressure within the ventricles and on the descending limb of the R-2 wave of the electrocardiogram, and reach their maximal amplitude during the isometric period. Our findings, however, indicate that in cases with split first sounds, the isometric contraction periods in the two ventricles are asynchronous and that the two ventricles, therefore, contribute main vibrations at different times.
}

the first sound in cases with normal mechanism the As-Vs time interval is one of the most important. (Margolies, unpublished observations.) Other conditions remaining the same, cases with short normal P-R intervals ( 0.12 to 0.14 second) tend to have sharp, loud first sounds, whereas cases with long normal $\mathrm{P}-\mathrm{R}$ intervals ( 0.18 to 0.21 second) tend to have dull, faint first sounds.

In two cases of complete heart block with split first sounds it was noted that the recorded vibrations of the two components during certain ranges of $\mathrm{P}-\mathrm{R}$ relation varied in amplitude independently of each other. In one case with varying $\mathrm{P}-\mathrm{R}$ intervals, due to ventricular escape, similarly marked variations in the two components of split sounds have been observed. In a case with complete heart block and a prolonged but not split first sound, variations were noted in the early and late parts of the prolonged sounds dependent on the As-Vs relationship. It is the purpose of this paper to present data obtained in these cases and to discuss their bearing on the nature of the duality of the first sound.

Case I. J. H., an aged inmate of the Moss Home of the Jewish Hospital, was found to have complete heart block, an intraventricular conduction defect (shown by the QRS complex having a duration of 0.14 second), widely split first heart sounds and varying loudness of the first sound from beat to beat. The relative contributions of the two components to the variations in loudness of the first sound could not be recognized with any degree of accuracy on auscultation.

In Figure 2, a continuous strip of tracing is reproduced illustrating $(a)$ the variations in amplitude of vibrations of the first sound in successive beats and $(b)$ the two positions at which major vibrations may occur. In Figure 3, representative beats have been selected from a continuous strip (except beat 5 which was selected from another tracing) and arranged in an order to show the influence of various As-Vs relationships on the sound components.

Analysis of the variations in amplitude of the major vibrations of the two sound components shows the following points: 1 . The variations are great, in this respect corresponding to marked variations in intensity of the sounds as determined by auscultation. 2 . The variations in the two 


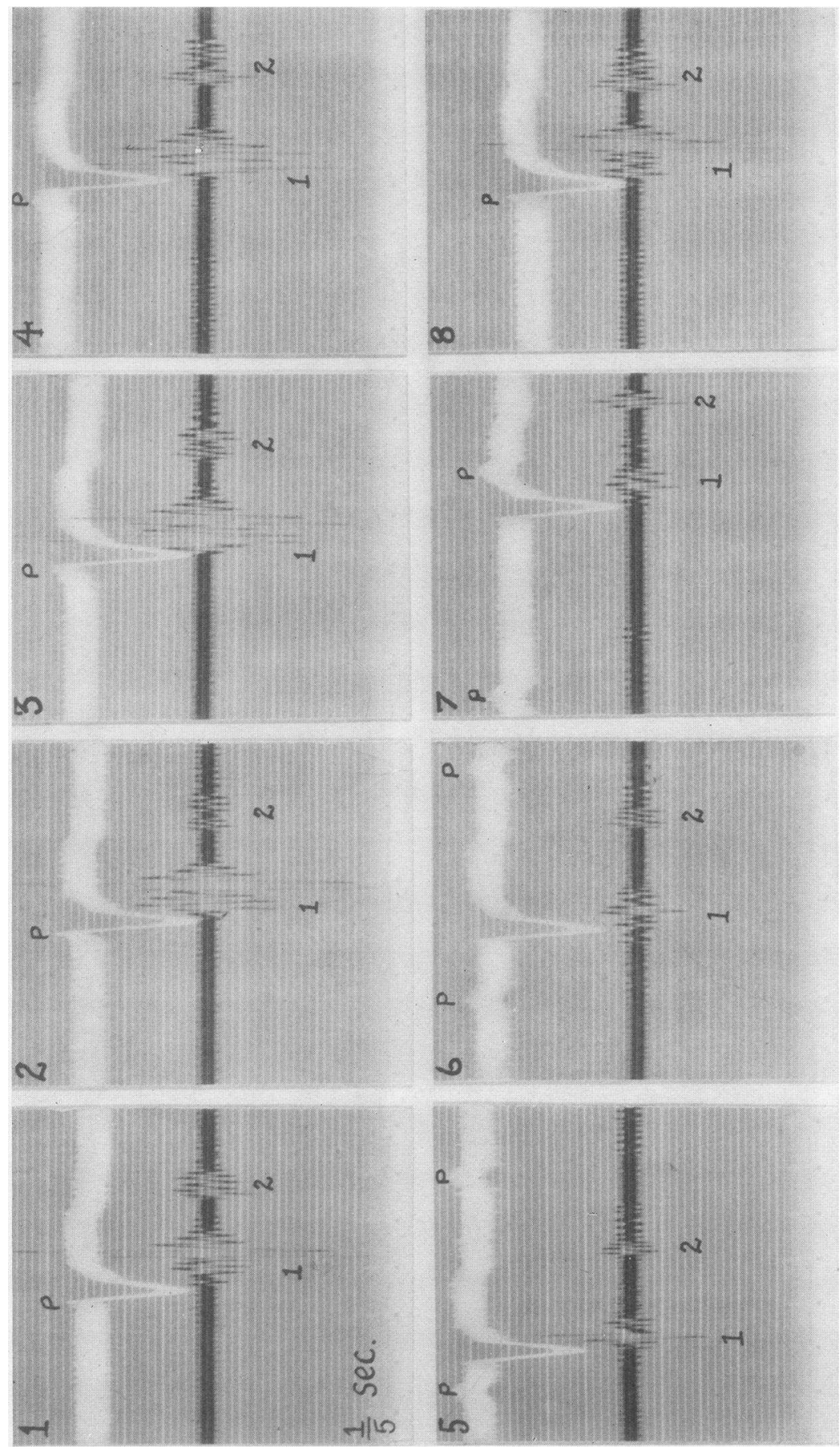

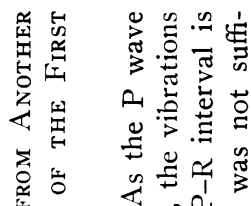

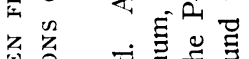

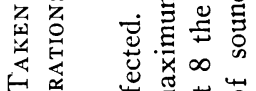

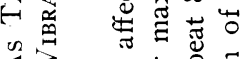

年>

了。 응 至要

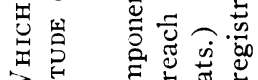

उ苛

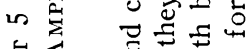

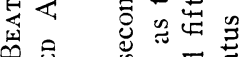

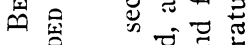

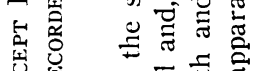

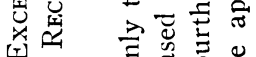

ब

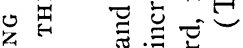

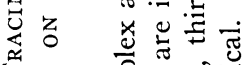

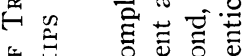

녕 ठ월

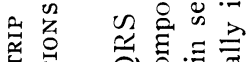

点安 0 व

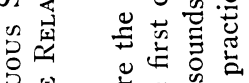

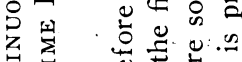

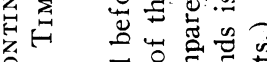

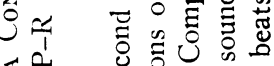

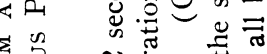

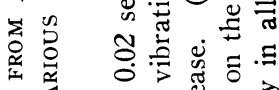

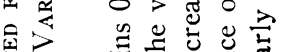

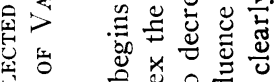

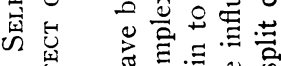

密

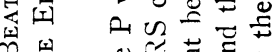

包舅苛节志

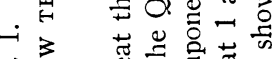

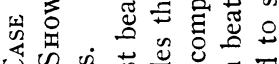

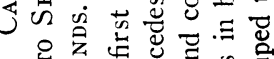

r

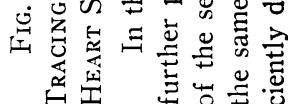


components are, to a certain extent, independent of each other. The following combinations occur:-both components may be recorded as having (a) small vibrations, $(b)$ large vibrations, (c) the first component may have vibrations of large or intermediate size and the second component small vibrations, $(d)$ the first component may have small vibrations and the second component vibrations of large or intermediate size. Thus practically all combinations of large and small vibrations may occur. 3 . The variations cannot be attributed to respiration since $(a)$ there is no correlation with the respiratory cycle and (b) variations continue when the breath is held.

The question must be considered as to whether the behavior of the two sound components described above could occur if both components of sound originated in the same ventricle. There are at least two reasons making such an explanation improbable.

1. Our previous work (6) showed that the first sound component falls during the isometric contraction phase of one ventricle. Thus, in Case 1, if both components originated in the same ventricle, the second component, which falls $0.10 \mathrm{sec}-$ ond after the first component, would have to occur well along in the course of ventricular contraction. Although under such circumstances it is quite conceivable that the second component might show variations dependent on As-Vs intervals, due to the effect of auricular activity on ventricular contraction, it is unreasonable to suppose that auricular contraction could influence the early part of ventricular contraction in one way and a later part of ventricular contraction in another way. In a discussion as to the various ways in which auricular contraction might influence ventricular behavior and consequently modify the first sound, we have pointed out that three factors might be involved: $(a)$ ventricular filling. (b) ventricular pressure and $(c)$ position of the $\mathrm{A}-\mathrm{V}$ valves (11). The time at which these factors may exert an effect must be at about the beginning of ventricular contraction. Thus, it seems necessary to assume, other phenomena remaining constant, that the character of a ventricular response must be determined at or before the time the first component of sound occurs. Under these circumstances it is theoretically pos- sible that the second component might vary (a) in the same direction as the first component, $(b)$ in the opposite direction or $(c)$ remain relatively constant. However, no rational explanation of independent variations based on the postulate that both components arise in the same ventricle occurs to us.

2. It was noted that changes in the second component of sound occurred when auricular contraction fell so near. the first component that it could scarcely have had a material influence upon the first component. Thus, in both Cases I and II (the latter to be reported below) auricular activity had no effect on the first component of sound when the $\mathrm{P}$ wave was buried in the QRS complex, although the second component in certain beats was materially influenced. As a matter of fact, in Case I the vibrations of the first component were not increased until the $\mathrm{P}-\mathrm{R}$ relation exceeded 0.03 second, and in Case II, not until the $\mathrm{P}-\mathrm{R}$ relation exceeded 0.05 second. In connection with these extremely short $\mathrm{P}-\mathrm{R}$ relations maximal amplitude of the vibrations of the second component occurred. In certain of the beats, in which only the vibrations of the second component were significantly increased, the $\mathrm{P}$ wave fell so late that auricular contraction could not have influenced the first component. Thus, in Figure 5, beat 7 (Case II) the $\mathrm{P}$ wave is completely buried in the QRS complex. Since the first sound component began 0.04 second after the beginning of the QRS complex, the beginning of the $\mathrm{P}$ wave could not have preceded the first component by more than 0.04 second. Taking into account the latent period of auricular muscle and the fact that ventricular contraction must have begun before sounds of ventricular origin could be recorded from the chest wall, it would appear that insufficient time was available for auricular contraction to have influenced the first component. As a matter of fact, apex cardiograms in this case showed no evidence of auricular activity until 0.06 to 0.07 after the beginning of the $\mathrm{P}$ wave; the summit of the wave due to auricular contraction fell 0.15 second after the beginning of the $P$ wave. The summit of this wave usually falls 0.08 to 0.14 second after the beginning of the $\mathrm{P}$ wave (12).

The above mentioned facts led us to reject the view that both main components of sound could 
have originated in one ventricle. Moreover, because of the evidence previously obtained suggesting that each ventricle contributes a component to split first heart sounds (based on the association of split first sounds and asynchronism in ejection), it seemed important to consider whether the variations in the two sound components were in accord with such a postulate.

It seemed to us that independent variation in the two sound components must be accounted for by equally independent effects of some factor or factors which influence production of sound. As indicated above, it is obvious on comparison of the variations of the two sound components with the duration of the $\mathrm{P}-\mathrm{R}$ interval that some $\mathrm{A}-\mathrm{V}$ relationship must be responsible for the variations in sound. The P-R interval, however, is a measurement of auriculoventricular conduction and, although it may serve to a certain extent as an indicator of the time relation of beginning auricular and ventricular contraction, it is not an accurate measurement of this relation. However, it is quite probable that a change in the $P-R$ interval reflects with reasonable accuracy a comparable change in the time relation of auricular and ventricular contraction. It cannot be the $\mathrm{P}-\mathrm{R}$ interval per se but the time relation of dynamic factors, the As-Vs interval, which influences the first sound. If there is present asynchronism in either auricular or ventricular contraction, there will be differences in As-Vs intervals on the two sides, depending in extent on the degree of asynchronism. Thus, if the $\mathrm{P}-\mathrm{R}$ interval in any beat has associated with it different $\mathrm{As}-\mathrm{Vs}$ intervals on the two sides of the heart, the effect af auricular contraction on the two ventricles will not be strictly comparable. For example, if one ventricle begins to contract at the summit of the wave of auricular filling, it is likely that the other ventricle will begin to contract on the ascending or descending slope of the wave, or possibly even before or after the wave.

If it were possible to oltain accurate measurements of As-Vs intervals on the two sides in patients, the problem would be greatly simplified. It occurred to us that the intervals between the beginnings of $\mathrm{P}$ waves and sound components might serve, at least to a certain extent, as indicators of the As-Vs intervals on the two sides of the heart. Such measurements fail to reflect asynchronism in the waves of ventricular. filling due to auricular contraction. However, if, as stated by Wiggers (8), main sound vibrations are associated with the rise of intraventricular pressure during the isometric contraction phase, it may reasonably be assumed that the interval between the two components should reflect with considerable accuracy asynchronism in the onset of the isometric contraction phase of the two ventricles. On the basis of these considerations the variations in the sound components were studied with respect to the time relation of each component to the preceding $\mathrm{P}$ wave.

The relation in Case I between the variations in amplitude of the two sound components and the interval from the beginning of the $\mathrm{P}$ wave to each sound component are shown in Table I. The table shows that there is actually nothing haphazard about the variations of the sound components, even though they do vary independently of each other. Each has its own clear cut definite relationship to the $\mathrm{P}$ wave. Furthermore, it is of interest to note that the time zone of intervals between $\mathrm{P}$ waves and sound components, during which waves of large amplitude and waves of small amplitude are recorded, differ but little for the two components. These findings are, therefore, in accord with the postulate that one component is produced in one ventricle and the other component in the other ventricle, and that the apparently independent variations are actually dependent on different As-Vs intervals on the two sides.

The objection may be raised that variations in major vibrations from beat to beat, even in a continuous strip of tracing, do not necessarily indicate variations in loudness of sounds, although intensity is supposed to vary as the square of amplitude times frequency. As stated above, we have paid considerable attention to this point in the study of first sounds and have found that the loudest sounds, as judged by auscultation, are recorded with major vibrations of widest amplitude. However, this correlation is of no particular inportance so far as our present analysis is concerned. The significance of the variations in amplitude of vibrations, considered as evidence as to whether both components of sound origi- 
TABLE I

The influence of various $A-V$ time relations on the two components of split first heart sounds. In each case the data shozen are obtained from a single continuous tracing.

\begin{tabular}{|c|c|c|c|c|}
\hline \multicolumn{3}{|c|}{ Time interval } & \multicolumn{2}{|c|}{$\begin{array}{l}\text { Maximum } \\
\text { amplitude of } \\
\text { sound vibrations }\end{array}$} \\
\hline $\begin{array}{c}\mathrm{P}-\mathrm{R} \text { or } \\
\mathrm{R}-\mathrm{P}\end{array}$ & $\begin{array}{l}\mathrm{P} \text { to } 1 \text { st sound } \\
\text { component }\end{array}$ & $\begin{array}{l}\mathrm{P} \text { to } 2 \text { nd sound } \\
\text { component }\end{array}$ & $\begin{array}{l}\text { 1st com- } \\
\text { ponent }\end{array}$ & $\begin{array}{l}\text { 2nd com- } \\
\text { ponent }\end{array}$ \\
\hline seconds & seconds & seconds & $m m$ & $m m$ \\
\hline
\end{tabular}

Case I

\begin{tabular}{c|c|c|r|r}
\hline $\mathrm{R}-\mathrm{P}$ & & & & \\
0.11 & $-0.06^{*}$ & 0.04 & 8 & 7 \\
0.09 & -0.04 & 0.06 & 10 & 9 \\
0.07 & -0.02 & 0.08 & 9 & 13 \\
$\begin{array}{c}\text { P buried in } \\
\text { QRS }\end{array}$ & & & 8 & 42 \\
& & & & \\
P-R & & & & \\
0.02 & 0.07 & 0.17 & 8 & 42 \\
0.03 & 0.08 & 0.18 & 9 & 48 \\
0.05 & $0.10 \dagger$ & 0.20 & 18 & 48 \\
0.07 & 0.12 & 0.22 & 39 & 38 \\
0.10 & 0.15 & 0.25 & 34 & 24 \\
0.11 & 0.16 & 0.26 & 21 & 22 \\
0.12 & 0.17 & 0.27 & 25 & 20 \\
0.16 & 0.21 & 0.31 & 21 & 5 \\
0.18 to 0.80 & 0.23 to 0.85 & 0.33 to 0.96 & 8 to 11 & 5 to 8 \\
\hline
\end{tabular}

Case II

\begin{tabular}{c|c|c|c|c}
\hline $\begin{array}{c}\text { P buried in } \\
\text { QRS }\end{array}$ & & & 9 & 7 \\
& & & 9 & 15 \\
& & & 11 & 14 \\
& & & 12 & 8 \\
& & & 13 & 12 \\
P-R & & & & \\
0.01 & 0.05 & 0.13 & 15 & 18 \\
0.02 & 0.06 & 0.14 & 14 & 20 \\
0.05 & 0.09 & 0.17 & 13 & 12 \\
0.06 & 0.10 & 0.18 & 22 & 10 \\
0.08 & 0.12 & 0.20 & 53 & 16 \\
0.09 & 0.13 & 0.21 & 51 & 15 \\
0.11 & 0.15 & 0.23 & 42 & 11 \\
0.14 & 0.18 & 0.26 & 16 & 7 \\
0.15 & 0.19 & 0.27 & 30 & 12 \\
0.18 to 0.55 & 0.22 to 0.59 & 0.30 to 0.67 & 6 to 14 & 6 to 13 \\
\hline
\end{tabular}

* The minus signs indicate that the $P$ wave followed rather than preceded the sound component.

† The numbers in heavy type indicate the time relations which are associated with significantly increased amplitude of sound vibrations.

nated in a single ventricle or one from each ventricle, remains the same whether the variations in amplitude reflect variations of intensity or some other quality of the sounds.
Case II. A young woman, 31 years old, had complete $\mathrm{A}-\mathrm{V}$ heart block, slightly increased intraventricular conduction time (duration of QRS complex 0.11 second), a widely split first heart sound and a variation in loudness of the first sound from beat to beat. In these respects the findings were similar to those of Case I, except that the duration of QRS was less. Numerous records of heart sounds were made, a sample of which is shown in Figure 4. In Figure 5, representative beats were selected from a continuous tracing to show the effect of various $A-V$ relations on the two components of the first sound. The measurements of amplitude of the largest vibration recorded for each sound component in a continuous strip of tracing are recorded in Table I, under Case II. The data are arranged with reference to the duration of the intervals between $\mathrm{P}$ waves and sound components, as in Case I. The variations in amplitude of vibrations of the first component were considerably greater than those of the second component, whereas in Case I the variations were slightly greater in the second component. However, the data in Case II (Table I) make it clear that in beats with relatively short As-Vs intervals the two components vary independently of each other. The zone of As-Vs intervals with maximum amplitudes of the first component is clearly displayed. Although the shortest As-Vs time intervals associated with maximal vibrations of the second component could not be clearly determined because the $\mathrm{P}$ wave was buried in the QRS complex, the longest intervals associated with maximal vibrations were nearly the same as in the case of the first component. The number of observations that could be made in a single strip, such as the one used, may be regarded as too few to be convincing. Similar data, however, were also obtained from other strips of tracing made at various times.

The variations in the amplitude of the major vibrations of the two components of the first sound with reference to their relations to $P$ waves in this case are of the same nature as those observed in Case I. It is, therefore, unnecessary to repeat the points made to justify the conclusion that one component originated in the left ventricle and the other in the right ventricle. 


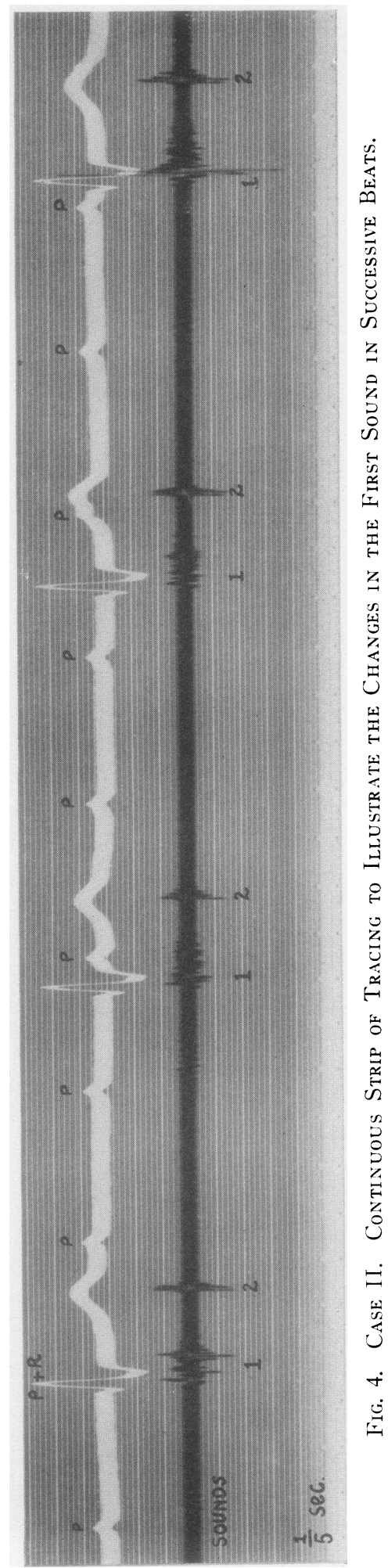

Case III. A man, 65 years old, had ventricular escape. Each cycle of the abnormal mechanism was terminated by an apparently premature ventricular beat. The apparently premature ventricular beat occurred whenever an auricular beat lagged far enough after the preceding ventricular beat to permit A-V conduction. The QRS complexes had a duration of 0.07 second, indicating the absence of intraventricular conduction defect. The first heart sound was found on auscultation to be split and also to vary markedly in loudness from beat to beat.

The sound tracing (Figure 6) shows that variations in amplitude of major vibrations of the two components of the first sound were, to a certain extent at least, independent of each other. Thus the amplitude might be (1) large in both components, (2) small in both, (3) large in the first and small in the second or (4) small in the first and large in the second. These various combination are similar to those found in Cases I and II. The tracing shows that marked variations in amplitude of vibrations are associated with changes in $\mathrm{P}-\mathrm{R}$ time intervals. No accurate analysis of this relation, however, can be made in Case III because of the fact that the ventricular arhythmia also influences the sounds (10). Nevertheless, the fact that the two components of sound are capable of varying independently of each other points, as in Cases I and II, to the postulate that one component originates in the left ventricle and the other component in the right ventricle.

The fact that in Case III there is no delay in intraventricular conduction, as in Cases I and II, to account for asynchronism in the early phases of ventricular contraction on the two sides, requires comment. In our previous study it was found that asynchronism in the beginning of ejection from the two ventricles occurred in cases with normal intraventricular conduction and split first sounds (6). Whether these differences in the ending of the isometric contraction phase (ejection) were due to inequality in its duration on the two sides or to asynchronism in onset or to both, could not be determined. The differences in the two components of sound depending on As-Vs intervals could be equally well accounted for on theoretical grounds by asynchronism in auricular systole on the two sides. The particular point we 

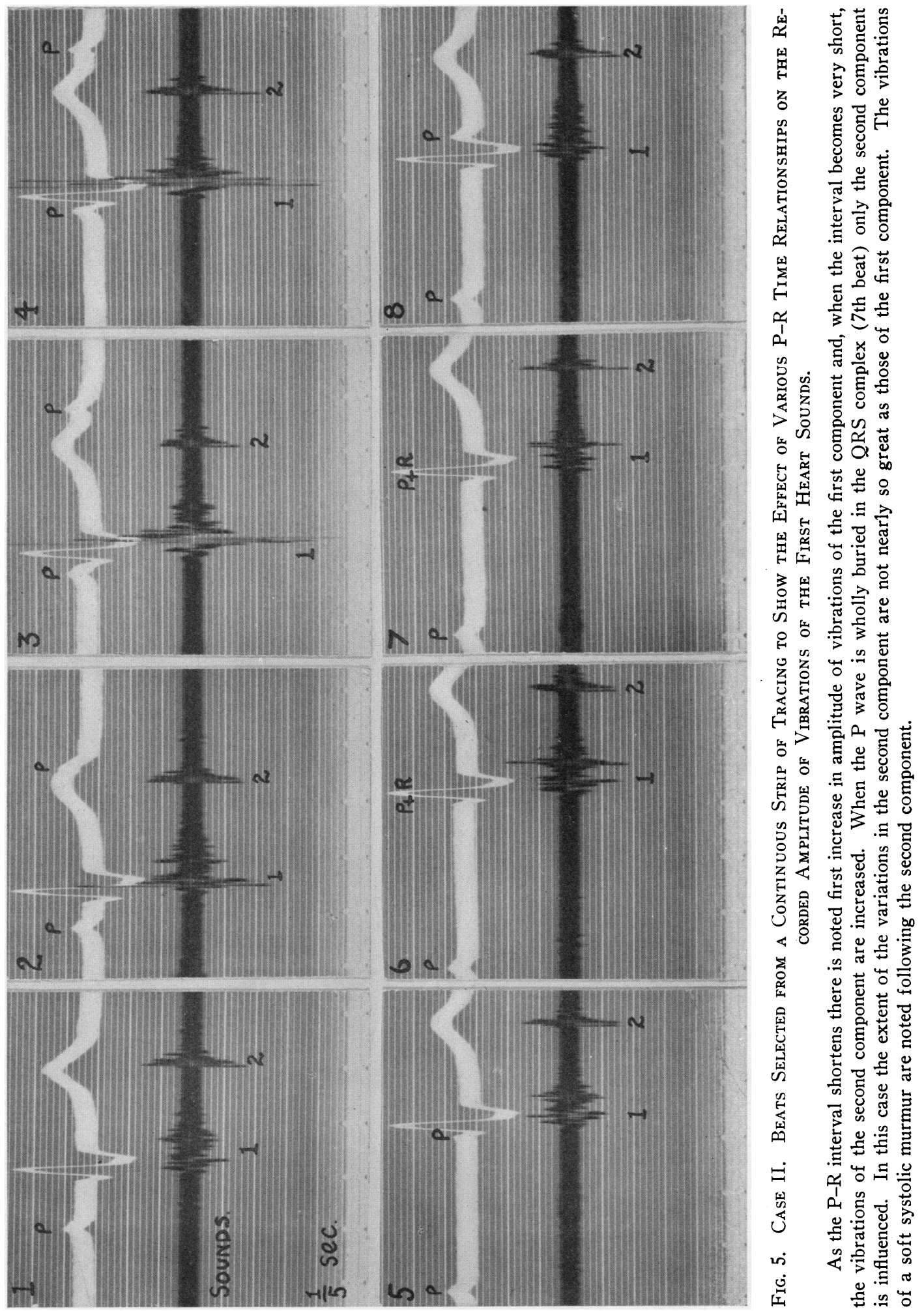


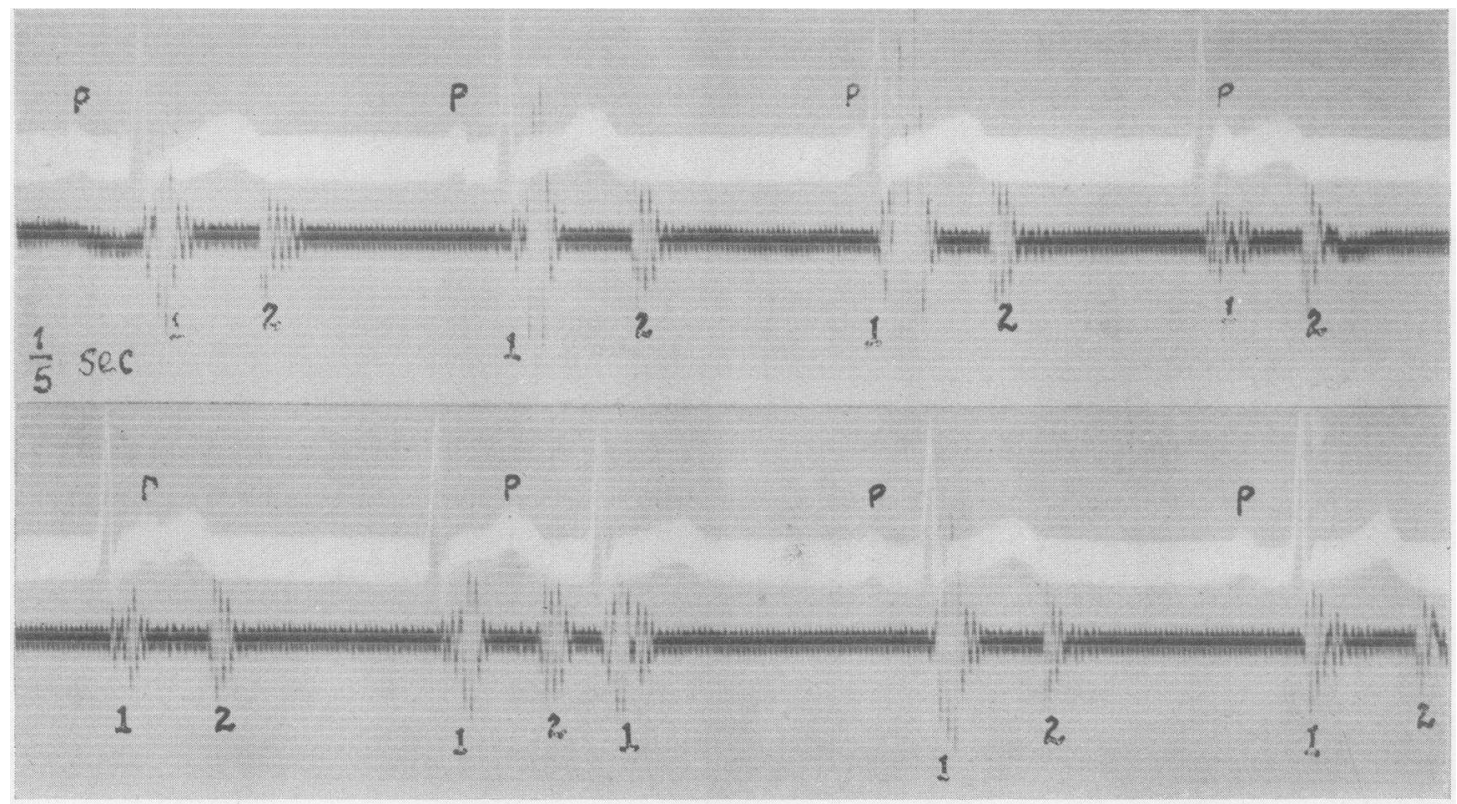

Fig. 6. Case III. The Lowrir Strip Is a Continuation of the Upper

The variations in the two components of the first sound, particularly those in the lower strip, are not entirely due to changes in $\mathrm{A}-\mathrm{V}$ time relation but are doubtless also influenced by the ventricular arhythmia.

wish to emphasize in this connection is that, although the mechanism responsible for the variations in sound components cannot be demonstrated completely by the studies thus far made, the occurrence of independent variations depending on changes of As-Vs intervals in cases without delay in the spread of intraventricular conduction is in no way inconsistent with the postulate that one component is contributed by each ventricle.

Case $I V$. A man, 55 years old, had complete heart block but no intraventricular conduction defect (duration of QRS complex 0.08 second). Auscultation revealed the fact that the first heart sound was prolonged but not definitely split and that there were variations in loudness of the sounds, certain beats being much louder than others. A representative strip of the sound tracing obtained in this case is shown in Figure 7. Marked variations in amplitude of major vibrations of the first sound are present, depending on the duration of the As-Vs interval. In this respect the behavior of the first sound corresponds with that observed in most other cases of complete heart block.

The sound tracing (Figure 7 ) shows, during a certain range of $\mathrm{P}-\mathrm{R}$ relations in which large vibrations occur, that as the interval shortens, the position of the largest vibrations shifts from the first part of the prolonged sound to the last part. This behavior is so similar to that observed in the two components of the split first sound in Cases I and II as to suggest that prolongation of the sound is due to slight asynchronism of the two components, not sufficient to cause actual separation from each other, and that the mechanism responsible for the variations is essentially the same as in Cases I and II.

\section{COMMENT}

All the facts available at this time appear to be consistent with the postulate that in split first heart sounds one major component is contributed by the right ventricle and the other major component by the left ventricle. The alternative view, that both components arise in the left ventricle or that both receive contributions from each ventricle, seems improbable, at least so far as the cases we have studied are concerned. Furthermore, there is some evidence that single first sounds are often contributed to by a component from each ventricle, better synchronized than in the case of the split sounds. It is, of course, probable that in certain 


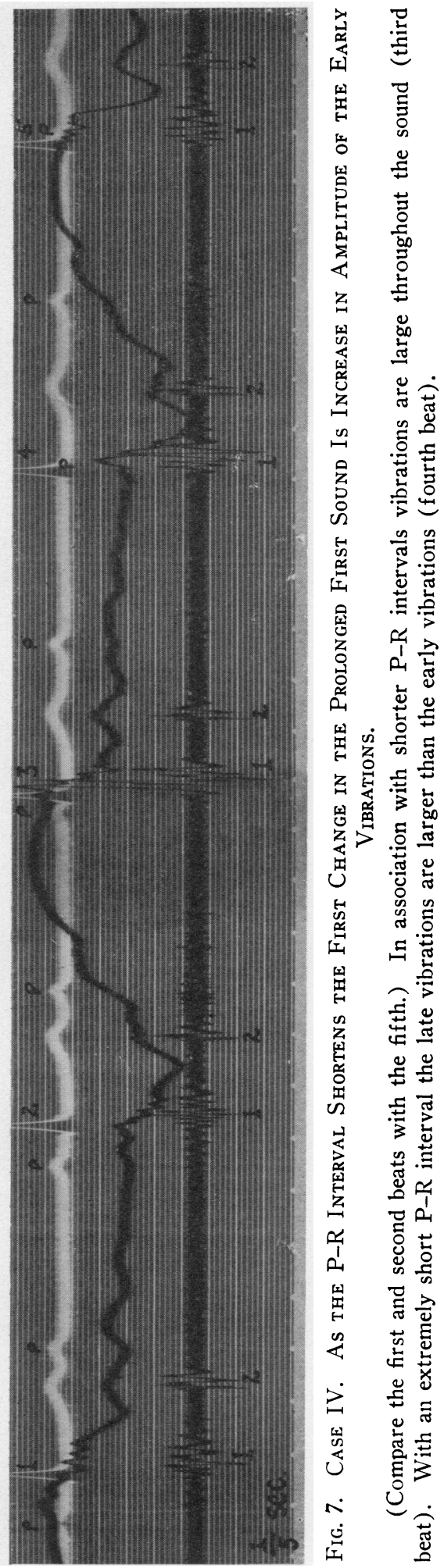

cases, possibly in many cases, the contribution from one ventricle may be negligible.

It is of interest that the duality of the first sound appears to be analogous in certain respects to that of the second sound. We have previously shown that (1) splitting of the second sound is associated with asynchronism in the ending of the ejection period from the two ventricles and must be due, therefore, as has been generally believed, to asynchronism in closure of the aortic and pulmonic valves (6); (2) each component is a short sharp sound; and (3) imperfect synchronization results in a prolonged second sound (13). It is, therefore, highly probable that in most cases with single second sounds both sides contribute a component to the sound. Furthermore, it is noteworthy that bundle branch block, with its marked asynchronism of the entire ejection phase, tends to be associated with rather wide splitting of both the first and second heart sound (6).

Our studies do not solve the problem as to how a sound component is produced in a ventricle, although they do show rather clearly that both sound components may be modified tremendously by relatively slight changes in As-Vs intervals. Furthermore, they indicate that in studies of the first sound the nature of the duality must be borne in mind, particularly in any investigation of the time relations of the sound. Failure to do so may result in serious error.

In view of the remarkable relation between the duration of As-Vs intervals and the loudness of the first sound, which is so clearly shown in dealing with single sound components, the recent contribution of Dock (14), regarding the mechanism of production of the first sound, requires comment. Dock has performed experiments on the $\mathrm{A}-\mathrm{V}$ valves which he believes support the view that the first sound is produced by vibration of these valves. The experiments, however, which brought about modification or disappearance of the first sound included drawing a ligature tightly around the $\mathrm{A}-\mathrm{V}$ groove, which apparently trapped blood within the ventricles but did not prevent them from "contracting vigorously." Thus, socalled isometric contraction with a volume of blood within the ventricles was obtained, the A-V valves being held presumably immobile by the ligature around the $\mathrm{A}-\mathrm{V}$ groove. 
In our opinion it is difficult to draw conclusions regarding the first sound from this interesting experiment. The production of sound requires the application of an appropriate force to a structure capable of vibrating within the frequency range of audibility. Therefore, if conclusions are to be drawn from experiments in which one source of vibrations is eliminated, it is necessary to control the application of force so that possible vibrations of other structures are not suppressed. Wiggers has pointed out that variations in systolic discharge and arterial resistance markedly influence production of sound (15). However, the fact illustrated in Table I of this paper, that a change in the $\mathrm{P}-\mathrm{R}$ interval of not more than 0.03 to 0.04 second is associated with change from large to small amplitude of sound vibrations without material change in the pulse volume, as shown by optically recorded pulse tracings, indicates clearly and significantly the delicate balance of factors concerned in production of heart sounds.

There are a number of facts which support the view that the factor initiating the major vibrations of the first sound is the rise of intraventricular pressure, which results from the force exerted by ventricular contraction on the ventricular content, during the isometric contraction phase. So far as we are aware, there is no evidence against such a postulate. Observations on patients with various levels of blood pressure suggest that the gradient of this rise of intraventricular pressure may be quite as important in production of sound as its extent, possibly even more so. Thus, some patients with low systolic blood pressure have louder first sounds than other patients with high systolic pressure. It seems quite possible that experiments of a type which presumally modify ventricular dynamics so much as is to be expected from ligature of the auriculoventricular groove, might modify to a considerable extent the gradient and the extent of the rise of intraventricular pressure. In view of the fact that, as has been demonstrated by the study of $\mathrm{As}-\mathrm{Vs}$ intervals, even slight changes may cause tremendous variations in sounds, it does not seem unreasonable to suppose that the disappearance of sounds, noted by Dock in the course of his experiment, may be clue at least in part to changes in ventricular dynamics rather than wholly to immobilization of the $\mathrm{A}-\mathrm{V}$ valves. Therefore, it does not appear to be established that immobilization of the valves per se is the importani factor in the suppression of the sounds. The question as to what anatomical structure or structures supply the most important vibrations during ventricular contraction remains, in our opinion, an unsolved problem.

We have provisionally adopted the following views regarding the first heart sound, subject to modification as a result of future investigation: (a) the important di:ality of the first sound is due to the contribution of both right and left ventricular components; (b) each component is produced during the ventricular isometric contraction phase and is associated with the period of rapidly rising pressure; $(c)$ factors which modify the curve of rising pressure will modify the sound. One of the most important of such factors is the wave of ventricular filling produced by auricular contraction, which, by influencing (1) intraventricular volume, (2) initial intraventricular pressure and possibly also (3) the position of the $\mathrm{A}-\mathrm{V}$ valves at the instant ventricular contraction begins, may exert a profound influence on sound production. Clinical observation indicates that the physiological state of the ventricles is also a factor. Thus, in hyperthyroidism the first sound tends to be loud; when the ventricular action is weakened, as in the common type of bundle branch block, acute coronary occlusion or a moribund state, the first sound tends to be faint. However, weakened ventricles, unable to maintain cardiac compensation, are frequently capable of contributing loud sounds. (d) The time relations of the isometric contraction phase in the two ventricles determines whether the first sound will be short, prolonged or split. It is probable, however, that certain first sounds are short because of failure of one ventricle to make a significant contribution to the sound. (e) Heart sounds are influenced by the physical properties of the structure or structures set into vibration and are modified by the physical properties of structures through which these vibrations are transmitted to the surface of the chest. It has not been determined what structure or structures contribute the most important sound vibrations. 


\section{SUM MARY}

1. Two cases are presented, both exhibiting complete heart block and split first heart sounds. Heart sound tracings showed marked variations in amplitude of vibrations in each component of the split sounds. During certain ranges of $P-R$ relations the variations in the two components appeared to be independent of each other.

2. Evidence previously obtained suggests that in cases with split first heart sounds there is asynchronism in the isometric contraction phase of the two ventricles, corresponding to the split between the two components. This affords a reasonable explanation for the variations in the two sound components, namely, that they are due to different As-Vs intervals on the two sides of the heart. Furthermore, the range of As-Vs intervals associated with increased amplitude of waves differs but little for the two components. Thus, the apparently haphazard variations in the second component are found to be of the same nature as the variations in the first component. Both components behave as do single first sounds when they are influenced by As-Vs intervals which change from beat to beat.

3. In a number of beats recorded, particularly in Case II, the $\mathrm{P}$ wave falls so late with reference to the first sound component that auricular contraction could not have materially influenced this component, although marked increase of amplitude in the waves of the second component occur.

4. In Case III (in which changes in As-Vs intervals are due to ventricular escape) independent variations in the two components of the first sound occur, which are similar to those observed in Cases I and II. This case is not as favorable for analysis as Cases I and II because the presence of ventricular arhythmia may also influence the first heart sound.

5. Case IV shows complete heart block and a prolonged first sound. During a certain range of $\mathrm{P}-\mathrm{R}$ relations the first part of the sound is represented by vibrations with large amplitude, and during a slightly shorter range the last part of the sound is represented by sounds with large amplitude. Since this behavior corresponds to that of the two components of a split first sound during certain ranges of $P-R$ intervals, it sug- gests that the prolongation of the first sound is due to slight asynchronism of the two major sound components.

6. All the findings in our cases are in accord with the postulate that one main component of the first sound is contributed by one ventricle and the other main component by the other ventricle. They lend no support to the view that both components arise in a single ventricle and, as a matter of fact, cannot be accounted for on such a basis.

7. It is suggested, on the basis of present knowledge, that major sound vibrations in each ventricle are associated with the period of rapidly rising pressure of that ventricle during the isometric contraction phase and that factors which modify the curve of rising pressure also modify production of sound. The volume of sound does not necessarily parallel the extent of rise of intraventricular pressure; the sounds are capable of varying independently of the output (as shown by optically recorded arterial pulse waves); it is probable that the gradient of rise of pressure is the most important factor. It has thus far not been determined what structure or structures contribute the main sound vibrations in response to the application of force initiated by the contraction of the ventricular muscle upon the ventricular contents.

\section{BIBLIOGRAPHY}

1. Laennec quoted from Bouillaud, J. P., Traité clinique sur des maladies du coeur. Baillière, Paris, 1835, Vol. I, p. 106.

2. King, J. T., Bundle branch block: A case analysis with especial reference to incidence and prognosis. (References to previous observations on reduplication are included.) Am. J. M. Sc., 1934, 187, 149.

3. Holt, E., Gallop rhythm. Collective review. (An excellent brief review of literature on reduplicated sounds is given.) Am. Heart J., 1927, 2, 453.

4. Bard, L., De la multiplicité anormale des bruits du coeur. Semana méd., 1908, 28, 3.

5. Wiggers, C. J., Modern Aspects of the Circulation in Health and Disease. Lea and Febiger, Philadelphia, 1923, pp. 319 and 313.

6. Wolferth, C. C., and Margolies, A., Asynchronism in contraction of the ventricles in the so-called common type of bundle branch block: Its bearing on the determination of the side of the significant lesion and on the mechanism of split first and second heart sounds. Am. Heart J., 1935, 10, 425. 
7. Katz, L. N., The asynchronism of right and left ventricular contractions and the independent variations in their duration. Am. J. Physiol., 1925, 72, 655.

8. Wiggers, C. J., Modern Aspects of the Circulation in Health and Disease. Lea and Febiger, Philadelphia, 1923, p. 315.

9. Griffith, T. W., Remarks on two cases of heart block. Heart, 1912, 3, 143.

10. Wolferth, C. C., and Margolies, A., Certain effects of auricular systole and prematurity of beat on the intensity of the first heart sound. Tr. A. Am. Physicians, 1930, 45, 44.

11. Wolferth, C. C., and Margolies, A., The influence of auricular contraction on the first heart sound and the radial pulse. Arch. Int. Med., 1930, 46, 1048.

12. Wolferth, C. C., and Margolies, A., Gallop rhythm and the physiological third heart sound. Am. Heart J., 1933, 8, 441.

13. Wolferth, C. C., and Margolies, A., The various types of "extra" heart sounds. M. Clin. North America, 1931, 14, 897.

14. Dock, W., Mode of production of the first heart sound. Arch. Int. Med., 1933, 51, 737.

15. Wiggers, C. J., Modern Aspects of the Circulation in Health and Disease. Lea and Febiger, Philadelphia, 1923, p. 323. 